\title{
On the Muricid snail, Plicopurpura pansa, in the Pacific coast of Central America
}

\author{
Hiroshi KITANI \\ Independent researcher, Japan \\ *Corresponding to: Hiroshi KITANI, Independent researcher, Japan; Email: hermosil123@yahoo.co.jp
}

Received: March 09, 2020; Accepted: March 15, 2020; Published: April 01, 2020;

\begin{abstract}
The Muricid snail, Plicopurpura pansa, in Central America has been studied, and the secretion of hypobanchial gland and its utilization are discussed. P. pansa exudes a few drops of secretion in the gland at being picked up. The secretion has been used historically in various forms for dyeing. There is no other region where the dyeing by drops has been carried out. It is noteworthy that the shell is not hammered or cracked, and the snail is released or put back at the same place where it was. The name of "Pacific purple" is proposed for the dye and its color. A decreasing the stock population and the smaller individuals in size are reported. The Pacific coast of Central America is a unique area of artisanal textile culture, and intensive investigations on the species are now required for biological and cultural conservation.
\end{abstract}

Keywords: Muricid, Plicopurpura pansa, Central America, purple dye, dyeing method, Pacific purple, secretion, hypobranchial gland, 6,6'-dibromoindigo

\section{Secretion of the gland and dyeing}

The present study of Plicopurpura pansa focuses on the secretion in hypobranchial gland and a utilization of that, and reviews the result of field studies in El Salvador. Little comparative study among the species in other regions was conducted.

The Muricid snails are characterized by the hypoblanchial gland, and its secretion makes it possible to dye textiles. The dying activities are reported historically in the Pacific coast of Central America and in Mediterranean region, especially at Phoenicia $[1,2]$. In European countries, the dye and its color has been called Tyrian purple, Royal purple, Phoenician purple, and so on $[2,4]$. The color pigment was revealed to be 6,6 -dibromoindigo, and the chemical structure is shown (Figure 1) [3].

The distribution of P. pansa in El Salvador is limited at headland area with breaking water and rocky shore, not sand beach or stone beach, and the dyeing activities have been carried out in there.

The present study groups the dying methods into the two (Figure 2). The one (A) is the method by the drops of secretion and the second (B) by the tissues cut out of the gland. The former has been introduced only in Central America.

It is observed that $P$. pansa exudes a white liquid of secretion at being picked up, which means that the white liquid flows out in the shell aperture, and it's volume is about $0.3 \mathrm{ml}$ or 3 drops per individual of $3 \mathrm{~cm} / \mathrm{shell}$ length. However no comparative study about the relation between the volume and the body weight/size was conducted, the volume of the secretion of $P$. pansa is supposed to be too much than that of other Muricid. It is a characteristic of the species, and other Muricid requires a cracking the shell and cutting the tissue out of the gland for extracting dye pigment.
The method by drops (A) is very efficient for dyeing directly. The present study confirmed a stocking of fresh drops in refrigerator for a few days, which is very convenient for laboratory works. At flowing out the white liquid into the aperture, it is possible then to drop the liquid and dye directly the cotton yarn in the hand. The color of drops changes gradually from milky white, white green, and finally to purple color in 15 minutes under the sunlight.

The second (B) requires a process of cutting the tissue out of hypobranchial gland. After grinding the tissue in a bowl to be a watery paste, it is now possible to use it directly for painting or dying textiles (B1). Furthermore, the cut-out tissue is applicable for extracting the dye by chemical reductant (B/B2, B3) (Figure 2). After obtaining the paste (B), an adding water makes it possible to filter the fluid, and then the dye pigment, 6,6'-dibromoindigo, is extracted in the liquid filtered. It is now possible to reduce the liquid by adding the reductant such as sodium hydrosulfite (Figure 1). Then, the dyeing textile is possible in a dark room and then after under the sunlight for coloring. In this case, a volume of textile can be dyed smoothly. Powdered dye is also available after drying the filtered liquid, and then the dyeing is introducible at any place and at any time under the same procedure of B1. The method by reduction (B) has not been introduced in Central America.

The dye and its color have been called in general Tyrian purple $[2,4]$, but it is considered to be a product by reduction. That product has not been introduced in Central America, neither Tyrian purple. The dyeing method by the pure secretion and that by reduction are not the same, however the color pigment 6,6'-dibromoindigo is the same among the species. The present study names it "Pacific purple" for the dye and its color originated from the drops of P. pansa, which shows a better distinctiveness of the dyeing. 


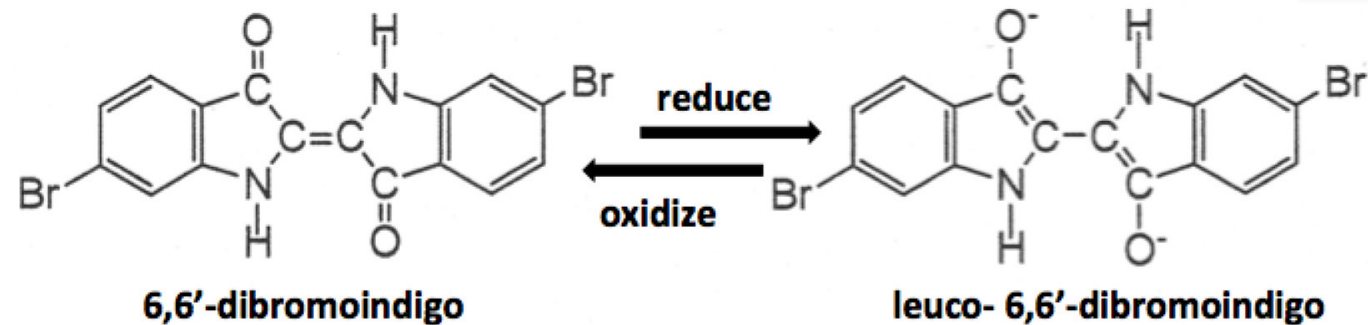

Figure 1. Chemical structure of 6,6'-dibromoindigo, and the process of reduction, leuco- 6,6'-dibromoindigo (Sawada 2014)

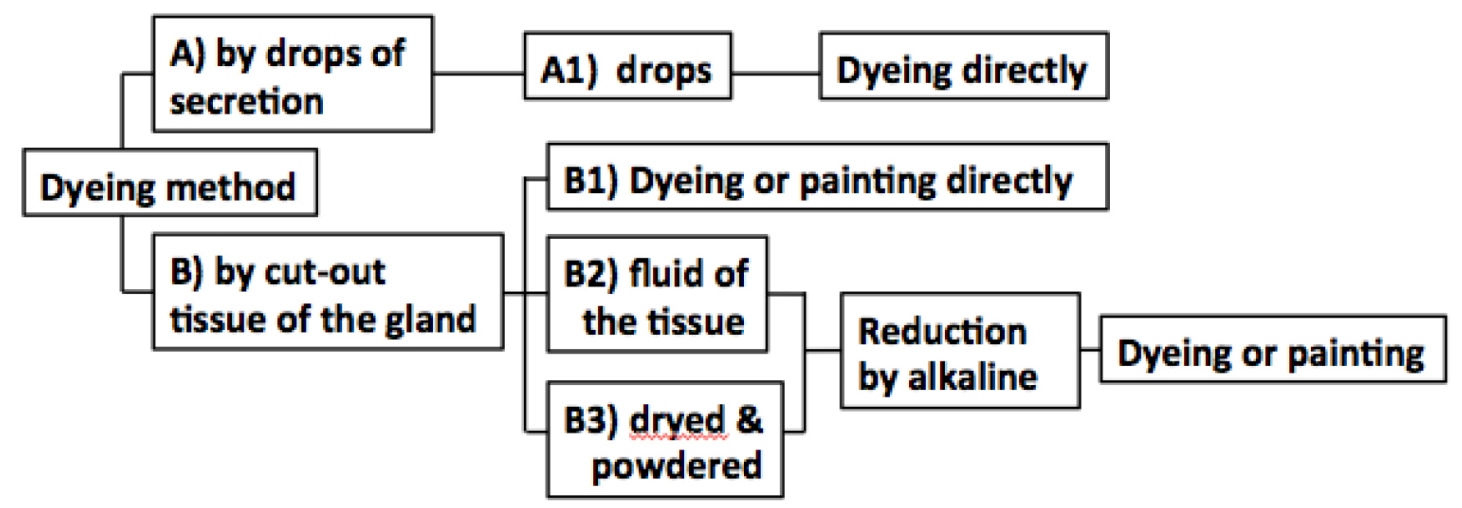

Figure 2. Dyeing methods by the secretion of Muricid snail

\section{Biology}

The functions of the secretion have been unclear. Some observations reported a toxic or anesthetic substance against the prey. Muricid such as oyster drills is a well known predator and a possible user of secretion to oysters. It is observed in laboratory that Rapana sp. uses the secretion against bivalves. The present study observed $P$. pansa preying on a smaller snail, Littorina $s p$.. It is experienced that the whole meat stimulates or irritates the tongue at eating and is inadequate for seafood, however some large sized snails such as Conchalepas sp. in Chile and Peru, and also Repana sp. in Turkey are important sea foods, but the secretion of these species are not utilized currently.

The habitat of $P$. pansa in El Salvador is limited on the rock surface or in a crack of rock, and stays for days at 3-5 m over the water line, where is a zone of without or less water. Video movies in Mexico show the collecting snails and dyeing at water line, which is a remarkable difference of the habitat. The behavior and habitat induce an assumption that the secretion would effective for a respiration while staying over the water line, because it is supposed that the water stocked inside the shell may be insufficient for staying days over the water line. It is supposed that Muricid in the water does not need additional oxygen, and secretes successively little volume of secretion. No conclusive study has been conducted on the secretion, but the present observation on $P$. pansa concludes that the releasing snails after dyeing should be returned to around the water line, not the same place where it was.
It is reported also that the Muricids lay eggs in capsules and it is reported that damaged capsules show a dark purple color, which suggests that the liquid in the capsule would contain the color pigment or the secretion. The biological study on egg capsule would be effective for larval production in laboratory.

There are little biological and ecological studies such as growth, reproduction, larval development, habitat, daily and seasonal movement, stock population, regional difference of the species, ad so on, which seem to be obstacles for the advanced developments and studies of $P$. pansa.

\section{Development and conservation}

Central America is the only place where the dyeing by secretion of Muricid is carrying out. It is a traditional culture for a long time [1], and the Pacific purple has been popular among the local people, however a limited production due to unstable dyeing works at seashore. No evidence of symbol color of authority or status has been reported as was Tyrian purple in Europe.

The dyeing textile by drops is very efficient and there would be no local need for improving the method or developing a new utilization. The present study has observed that a general interest in the natural purple is fading away, but a high interest exists also in enjoying the dye and its color by different ways from the past, specially in mysterious color changing every second, saying "palm-top miracle and wonder".

The present study suggests new utilizations such as a body painting, nail art, painting on the T-shirts, tourisms, traditional 
dyeing, biological/chemical study for younger generations, as well as a possible pharmaceutical and cosmetic use, and so on. The chemical product of 6,6'-dibromoindigo has been developed synthetically in Japan and it is reported that the examination of the fatness such as rubbing, washing, perspiration, and against light shows a better grade than those of indigo [3].

There seems to be little technical obstacles for utilizing the drops, but exists biological obstacles mentioned previously. Furthermore, some articles $[1,5]$ reported a possible fade-out of the culture and a heavy exploitation of the resources in the past.

The textile culture in Central America is very unique, and the tradition of living together with the resources of Pacific purple is highly praised. The present study emphasizes the necessity of effective biological and cultural conservation programs for the regional assets before being forgotten.

\section{References}

1. Secretaría de Educación Pública(SEP) (1988) El caracol púrpura, Una tradición Milenaria en Oaxaca, Mexico,ISBN 968-29-1867-7 (in Spanish)

2. Takako Terada (2005) Fieldwork on shellfish purple, Kwassui bulletin 48, p51-61. Kwassui Women's University. (in Japanese)

3. Tadanobu Sawada, Hiroyuki Ishii, Harue Senou, Toyotoshi Ueda (2014) Color Fastness of Ancient Purple 6,6'-Dibromoindigo after Dyeing. The Journal of Silk Science and Technology of Japan. 22: 57-63.

4. Ludwig CA, Naegel, Federico A. Garcia-Dominguez (2006) Reproductive cycle of the purple snail Plicopurpura pansa (Gould 1853) from two locations at Baja California Sur, Mexico. Journal of Shellfish Research 25: 925-933 